\title{
TEST DE SUDOR EN RANGO INTERMEDIO: DESAFÍO DIAGNÓSTICO
}

\author{
INTERMEDIATE SWEAT CHLORIDE VALUES: A DIAGNOSTIC CHALLENGE
}

\author{
Dr. Pablo R. Jorquera P. \\ Pediatra Especialista en Enfermedades Respiratorias \\ Unidad de Broncopulmonar Infantil \\ Complejo Asistencial Sótero del Río
}

\begin{abstract}
INTERMEDIATE SWEAT CHLORIDE VALUES: A DIAGNOSTIC CHALLENGE
There is a group of patients with sweat test values at intermediate range $(30-59 \mathrm{mmol} / \mathrm{I}$ chloride) whose diagnosis is difficult to be classified, especially after the introduction of neonatal screening for cystic fibrosis in some countries. This has introduced new terminology and panels of experts from the United States and Europe have created guidelines for the evaluation and management of these individuals. There are few studies on the evolution of these patients, however all of them agree on a more benign evolution than for those who have altered sweat test (sweat chloride higher to $60 \mathrm{mmol} / \mathrm{l})$. The clinical monitoring is essential to obtain a proper diagnosis.
\end{abstract}

Keywords: sweat test, cystic fibrosis transmembrane conductance regulator

RESUMEN

Existe un grupo de pacientes con valores de test de sudor en rango intermedio (30-59 mmol// de cloro) cuyo diagnóstico es difícil de catalogar, especialmente luego de la introducción en algunos países del tamizaje neonatal para fibrosis quística. Se ha introducido nueva terminología y paneles de expertos de Estados Unidos y Europa han creado guías para la evaluación y manejo de estos individuos. Existen escasas descripciones sobre la evolución de estos pacientes aunque coinciden en una evolución más benigna que en aquellos que tienen test de sudor alterado (cloro mayor a $60 \mathrm{mmol} / \mathrm{l})$.El seguimiento clínico es fundamental para llegar a un diagnóstico adecuado.

Palabras clave: prueba del sudor, regulador de conductancia de transmembrana de fibrosis quística

\section{INTRODUCCIÓN}

La Fibrosis Quística (FQ) es una de las enfermedades que más comúnmente compromete la vida. Su incidencia varía según el grupo étnico: en Estados Unidos se calcula una incidencia de 1/3200 nacidos vivos en caucásicos, 1/15000 en afroamericanos y 1/31000 entre americanos de origen asiático (1). En Chile se estima una incidencia de 1/8000 a 1/10000 recién nacidos vivos (2).

Es en esencia una enfermedad genética autosómica recesiva caracterizada por la alteración del canal regulador de transmembrana de la Fibrosis Quística (CFTR), determinando una falla en el transporte de electrolitos través de la membrana celular epitelial (3). Se han descrito alrededor de 1700 mutaciones y polimorfismos del gen CFTR asociados a un amplio espectro de fenotipos.

La prueba diagnóstica gold standard es el test de sudor, considerándose como diagnóstico valores de Cloro mayores $60 \mathrm{mmol} / \mathrm{l}$, valores intermedios entre $30-59 \mathrm{mmol} / \mathrm{l}$ en menores de 6 meses y entre $40-59 \mathrm{mmol} / \mathrm{l}$ a partir de los 6 meses, y se consideran normales valores menores a $30 \mathrm{mmol} / \mathrm{l}$ en menores de 6 meses y menores de $40 \mathrm{mmol} / \mathrm{l}$ a partir de los 6 meses $(1,4,5)$.

Clásicamente el cuadro clínico se caracteriza por

\section{Correspondencia:}

Dr. Pablo R. Jorquera P.

Unidad Broncopulmonar Infantil

Complejo Asistencial Sótero del Río

Avenida Concha y Toro 3459, Puente Alto

Santiago, Chile.

Correo electrónico: pablojorquera@vtr.net enfermedad pulmonar crónica, insuficiencia pancreática, malabsorción intestinal, malnutrición, ileo meconial, sinusitis y poliposis nasal. Existen sin embargo individuos que presentan síntomas menos severos o disfunción en un solo sistema; estos pacientes pueden permanecer sin diagnóstico por años, incluso diagnosticarse en la edad adulta, pudiendo tener o no valores elevados de test de sudor $(1,6)$. Por otro lado el tamizaje neonatal, el cual permite realizar el diagnóstico en niños aún asintomáticos, introduce una nueva complejidad y en ocasiones un dilema diagnóstico: los recién nacidos con pruebas anormales de tamizaje pero con resultados no concluyentes en test del sudor y/o estudio genético (7)

Esta situación ha introducido una nueva terminología. El presente artículo tiene como objetivo presentar la nomenclatura actualmente utilizada y describir la información existente con respecto a la evolución de los pacientes con test del sudor en rango intermedio.

\section{NOMENCLATURA}

- $\quad$ Fibrosis Quística No Clásica: enfermedad similar a $F Q$ en por lo menos un sistema de órganos y falla en detectar las 2 mutaciones (4).

- $\quad$ Fibrosis Quística Atípica: usualmente tienen una mutación severa y otra menos frecuente, o repetición de trinucleótidos en el otro gen. Es una forma leve de FQ, pueden tener disfunción en un solo órgano, las manifestaciones clínicas pueden variar en el tiempo y el test del sudor puede o no estar alterado (6).

- $\quad$ Sindrome Metabólico Asociado a CFTR (CRMS): 
lactantes con niveles elevados de IRT (tripsinógeno inmunoreactivo) pero con test de sudor y estudio genético no concluyentes. Este término debe ser usado para un niño asintomático y con hipertripsinogenemia, en 2 situaciones:

con cloro entre 30-59 en menores de 6 meses o 40-59 mmol/l en mayores de 6 meses en al menos 2 ocasiones con 001 mutación positiva

concentración de cloro menor a $30 \mathrm{mmol} / \mathrm{l}$ en menores de 6 meses y menores de $40 \mathrm{mmol} / /$ a partir de los 6 meses con 2 mutaciones del CFTR en trans, en que no más de una se conoce por ser causante de enfermedad $(7,8)$.

Aunque claramente no se trata de una enfermedad metabólica, y no fue aceptada en Europa, el objetivo de esta denominación es lograr introducirla en la clasificación internacional de enfermedades (7).

- $\quad$ Tamizaje positivo para Fibrosis Quística, Diagnóstico no concluyente (CFSPID: Cystic Fibrosis Screen Positive, Inconclusive Diagnosis): término utilizado en Europa para los casos de lactantes con test de sudor normal $(<30 \mathrm{mmol} / \mathrm{l})$ y 2 mutaciones CFTR, por lo menos una de ellas con consecuencias fenotípicas inciertas, o con test de sudor en rango intermedio con hallazgo de una o ninguna mutación del CFTR (7).

- $\quad$ Fibrosis Quística de diagnóstico tardío: son los pacientes que inicialmente tenían valores intermedios en el test del sudor y que posteriormente cumplen criterios diagnósticos de FQ. Debido a la existencia de estos casos se debe destacar la importancia del seguimiento de los niños con valores intermedios $(7,8)$.

\section{CARACTERÍSTICAS CLÍNICAS DE PACIENTES CON TEST DE SUDOR INTERMEDIO}

Los datos clínicos de pacientes con test de sudor intermedio son limitados (8). Pueden haber pacientes que desarrollan síntomas mínimos, otros que desarrollan síntomas relacionados con el defecto del CFTR en órganos no respiratorios (ej: pancreatitis, infertilidad masculina) (3) y otros son tardíamente diagnosticados formalmente como $\mathrm{FQ}(8,9)$. Un elemento que hace difícil caracterizar este grupo de pacientes es el subdiagnóstico, confundiéndose con otras enfermedades del aparato respiratorio como el asma.

Gobau et al (10) reportan las características fenotípicas de pacientes europeos con test de sudor con valor intermedio. Es un estudio multicéntrico retrospectivo que compara 4 grupos: 59 pacientes con disfunción CFTR, 46 con valores de test de sudor en rango intermedio pero sin disfunción de CFTR (FQ improbable), 103 pacientes con $F Q$ y suficiencia pancreática y 62 pacientes con $F Q$ e insuficiencia pancreática. Al comparar pacientes con disfunción CFTR con aquellos con FQ improbable los primeros tenían mayor proporción de dedos en palillo de tambor, infecciones respiratorias recurrentes y aislamiento de Pseudomona aeruginosa y Estafilococo dorado. Cuando se comparó los pacientes con disfunción CFTR con aquellos con diagnóstico de $F Q$ y suficiencia pancreática los primeros tenían menos colonización por Pseudomona aeruginosa, mayor mediana de VEF 1 (89 vs 83\%), también era menos frecuente el ileo meconial, el síndrome de obstrucción intestinal distal y la enfermedad hepática.
Ren et al (11) describen en forma retrospectiva las características de pacientes con CRMS diagnosticados luego de tamizaje neonatal en un centro de referencia en Nueva York entre Ios años 2002 a 2010. Identifican 12 pacientes y los comparan con 27 pacientes diagnosticados con fibrosis quística en el mismo periodo. Ninguno de ellos presentaba insuficiencia pancreática y tenían menos cultivos positivos para Pseudomona aeruginosa. El número de pacientes con exacerbaciones respiratorias que requirieron hospitalización durante el periodo fue menor en el grupo con CRMS.

Groves et al (8) revisan en forma retrospectiva la experiencia de 15 años (1996-2010) de tamizaje neonatal en un centro de referencia de Sydney, Australia, identificando durante el periodo 29 pacientes con tamizaje positivo que presentaron test de sudor en rango interrmedio, 14 sujetos (48\%) fueron posteriormente diagnosticados como FQ ya sea por elevación de los niveles de cloro, pruebas de insuficiencia pancreática o infección respiratoria recurrente por Pseudomona o Estafiloco dorado, siendo todos estos pacientes heterocigotos para deltaF508. Al comparar estos pacientes con los 28 sujetos con FQ diagnosticados durante el periodo de tamizaje se observó una mejor función pulmonar a los 10 años y menor incidencia de aislamiento de Pseudomona aeuoginosa no mucoide, sin evidenciar diferencias en el estado nutricional ni en el aislamiento de Estafilococo dorado. Este estudio sin embargo muestra una mayor proporción que la observada en un estudio realizado en Victoria, USA, en el cual se diagnostica la enfermedad en 4/27 (15\%), correspondiendo a FQ de diagnóstico tardío $(8,9)$. Lamentablemente este estudio no describe seguimiento.

\section{EVALUACIÓN Y MANEJO INICIAL DE PACIENTES CON SCREENING NEONATAL POSITIVO Y TEST DE SUDOR DE VALOR INTERMEDIO}

El tamizaje neonatal identifica niños con alto riesgo de fibrosis quística (12). Aquellos que tienen test de sudor con cloro mayor a $60 \mathrm{mmol} / \mathrm{l}$ o 2 mutaciones de CFTR causantes de fibrosis quística no provocan problema diagnóstico, a diferencia de los casos cuyos valores de cloro están entre 30-59 mmol /l. Para orientar la evaluación y manejo de estos niños tanto en Estados Unidos como en Europa se ha intentado protocolizar los controles, exámenes y medidas terapéuticas $(12,13)$.

El año 2009 el panel de expertos de Estados Unidos propone guías para el manejo de niños con Sindrome Metabólico relacionado a CFTR (CRMS) (12). Estas guías recomiendan que al paciente con tamizaje neonatal positivo, con test de sudor realizado entre las 2 a 4 semanas de edad, con resultado entre 30-59 $\mathrm{mmol} / \mathrm{l}$ de Cloro, este examen se debe repetir a los 2 meses. En muchos casos se define el diagnóstico, pero si el valor permanece en rango intermedio se debe realizar estudio genético, evaluación clínica y consejo genético en un centro de FQ. Debiera realizarse un tercer test de sudor a los 6 meses (12). En el centro de FQ se recomienda que la evaluación sea en un lugar físico separado de los pacientes con diagnóstico confirmado con objeto de disminuir el riesgo de infecciones cruzadas. Además se sugiere toma de cultivo orofaríngeo y pruebas de medición de la suficiencia pancreática incluida elastasa fecal. No se recomienda de rutina realizar hemograma, pruebas hepáticas, albumina y niveles de vitaminas A y E; la radiografía de tórax es recomendada sólo si hay síntomas respiratorios. No se recomienda en forma rutinaria suplementos de 
vitaminas, como tampoco medidas de clearence respiratorio. Los niños que persistan asintomáticos deben ser controlados por lo menos 2 veces al año hasta los 2 años y una vez al año a partir de esa edad (12).

El año 2009 un grupo de expertos pertenecientes a diversos países europeos también generó un consenso para la evaluación u manejo de pacientes con tamizaje positivo y test de sudor con valores de Cloro entre 30-59 mmol // (13). El consenso europeo recomienda que los niños con tamizaje positivo y test de sudor con valores en rango intermedio sean evaluados en un centro especializado en $F Q$ donde debe repetirse el test de sudor. Si es normal el individuo no requiere más evaluaciones, sin embargo si repite el resultado intermedio y presenta una o ninguna mutación al panel local, debe realizarse un análisis genético extendido. Se recomienda realizar a todos estos niños elastasa fecal, cultivos de vía aérea y radiografía de tórax, pero otros exámenes más complejos (como tomografía computada y broncoscopía) estaría indicada sólo si la situación clínica lo amerita. Si no hay evidencia clínica de FQ el test de sudor debe ser repetido entre los 6 a 12 meses de edad. Si aparece evidencia clínica de la enfermedad o del defecto en el transporte de iones debe continuar seguimiento regular en el centro especializado. Aquellos individuos en quienes el resultado del test de sudor permanezca intermedio y no tengan evidencia clínica de enfermedad debería ser considerado para investigación dirigida al defecto fisiológico: potencial nasal, biopsia rectal o de intestino delgado para medir transporte transepitelial de iones. Sin embargo el consenso advierte que ninguno de estos métodos posee la validez del test de sudor.

\section{DISCUSIÓN}

Desde la introducción de la metodología estandarizada del test de sudor la definición de normalidad, valores intermedios y anormalidad ha sido aplicada universalmente (5). Estos valores se basaron en 7200 test de sudor realizados entre 1959 y 1966 , determinando un rango que si bien tiene limitaciones ha probado ser satisfactorio ya que según el registro de pacientes de la Fundación de Fibrosis Quística del año 2005 sólo un 3,5 \% de los pacientes diagnosticados con $\mathrm{FQ}$ en Estados Unidos tenían test de sudor con cloro menor a $60 \mathrm{mmol} / \mathrm{l}$ y 1,2 \% bajo $40 \mathrm{mmol} / \mathrm{l}$ (5).

Estos valores sin embargo no son representativos en neonatos y lactantes pequeños lo cual es de alta trascendencia considerando que al contarse con un programa de tamizaje neonatal se requiere de un diagnóstico adecuado. El reporte del comité de consenso recomienda que para menores de 6 meses los valores menores o iguales de $29 \mathrm{mmol} / \mathrm{l}$ hacen el diagnóstico poco probable, mayor o igual a $60 \mathrm{mmol} / \mathrm{l}$ son diagnósticos y entre 30-59 $\mathrm{mmol} / \mathrm{l}$ se consideran en rango intermedio, agregándose la recomendación que estos pacientes sean derivados a un centro especializado en fibrosis quística y el test de sudor sea repetido (5).

Al aplicar las guías de diagnóstico mencionadas anteriormente, muchos pacientes, incluso luego de repetido el test de sudor y realizado estudio genético persisten sin poder ser diagnosticados con FQ pero tampoco podemos considerarlos como sanos. Nos encontramos entonces frente a la necesidad de considerar una nueva categoría dentro del espectro de severidad de la enfermedad. La Figura 1 describe la sobreposición entre algunas de las categorías diagnósticas mencionadas (14).
Figura 1. Espectro de manifestaciones clínicas de la fibrosis quística

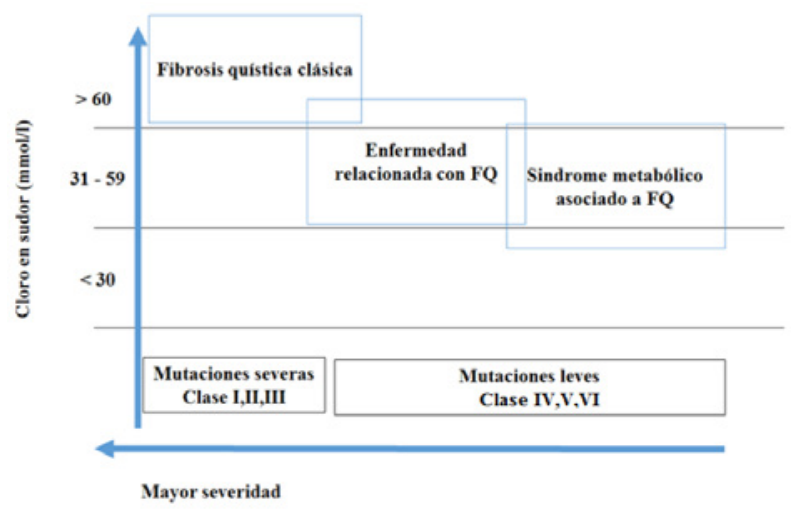

Defecto en mutaciones severas:

Clase I: síntesis de CFTR

Clase II: procesamiento de CFTR

Clase III: apertura canal

Defecto en mutaciones leves

Clase IV: conductancia del canal

Clase V: disminución producción CFTR

Clase VI: aumento eliminación CFTR en superficie celular

La variedad de definiciones y el escaso número de trabajos relacionados con este grupo específico de pacientes hace difícil hacerse una correcta idea de la prevalencia de esta situación y de sus consecuencias clínicas. Es probable que exista subdiagnóstico ya que los datos disponibles corresponden fundamentalmente a países donde se han instalado programas de diagnóstico prenatal. Algunos autores han mostrado que en pacientes con tamizaje neonatal positivo la relación fibrosis quística / CRMS sería de 2/1 (15).

En Chile por el momento no se cuenta con una conducta única para pacientes con test de sudor en valores intermedios, definir su evaluación y manejo será un importante desafío a medida que el tamizaje neonatal se extienda y sea garantizado como un derecho. Es necesario crear guías y consensos validados por la experiencia nacional y luego una adecuada difusión. En países donde se cuenta con claras guías al respecto, se ha demostrado un bajo cumplimiento de estas, como mostró un estudio realizado en Estados Unidos en 77 centros de referencia de FQ donde se evidenció gran variabilidad en procedimientos de test de sudor, niveles diagnósticos y terminología aplicada para estos pacientes (16).

Debemos tener presente que existen casos de difícil definición, especialmente en lactantes asintomáticos con tamizaje positivo, en ellos aún en ausencia de un consenso para su manejo, el seguimiento cuidadoso para detectar cambios clínicos es fundamental para llegar a un diagnóstico correcto.

En esta edición, Parra y cols describen un caso clínico de $F Q$ atípica, complementando la información del presente artículo (17).

El autor declara no presentar conflicto de intereses 


\section{REFERENCIAS}

1. Paranjupe S, Zeitlin P. Atypical Cystic Fibrosis and CFTRRelated Diseases. Clinic Rev Allerg Immunol 2008; 35:116123

2. Grupo Técnico, Programa de Fibrosis Quística Unidad de Salud Respiratoria, Subsecretaría de Redes Asistenciales Ministerio de Salud, Programa Nacional de Fibrosis Quística Orientaciones Técnicas Programáticas para Diagnóstico y Tratamiento. 2012

3. Bombieri C, Claustres M, De Boeck K, Derichs N, Dodge J, Girodon E.et al . Recommendations for the classification of diseases as CFTR-related disorders. Cyst Fibros 2011; 10 suppl 2: s86-s102

4. Bush A. Diagnóstico de fibrosis quística. Lo fácil, lo difícil, lo imposible. Neumol Pediatr 2010; 5: 15-22

5. Farrell $P$, Rosenstein $B$, White $T$, Accurso $F$, Castellani $C$, Cutting $G$ et al. Guidelines for Diagnosis of Cystic Fibrosis in Newborns Through Older Adults: Cystic Fibrosis Foundation Consensus Report. J Pediatr 2008; 153:s4-s14

6. Schram C. Atypical Cystic Fibrosis . Identification in the primary care setting. Can Fam Physician 2012 ;58:13411345

7. Levy H, Farrell P. New Challenges in the Diagnosis and Management of Cystic Fibrosis. J Pediatr 2015; 166:13371340

8. Groves T, Robinson P, Willey V, Fitzgerald D. Long Term Outcomes of Children with Intermediate Sweat Chloride Values in Infancy. J Pediatr 2015;166:1469-1474

9. Massie J. Clements B and the Australian Paediatric Respiratory Group. Diagnosis of Cystic Fibrosis After Newborn Screening: The Australasian Experience-Twenty Years and Five Million Babies Later: A Consensus Statement From the Australasian Paediatric Group. Pediatr Pulmonol
2005; 39: 440-446

10. Goubau C, Wilschanski, Skalincka V, Lebecque P, Southern $\mathrm{K}$, Sermet I et al. Phenotypic characterization of patients with intermediate sweat chloride values: towards validation of the European diagnostic algorithm for cystic fibrosis. Thorax 2009; 64: 683-691

11. Ren C, Desai H, Platt M, Dixon M. Clinical Outcomes in Infants With CysticFibrosis Transmebrane Regulator (CFTR) Related Metabolic Syndrome. Pediatr Pulmonol 2011; 46: 1079-1084

12. Borowitz D, Parad R, Sharp J, Sabadosa K, Robinson K, Rock $M$ et al. Cystic Fibrosis Foundation Practice Guidelines for the Management of Infants with Cystic Fibrosis Transmembrane Conductance Regulator-Related Metabolic Syndrome during the First Two Years of Life and Beyond. J Pediatr 2009; 155: s $106-s 116$

13. Mayell S, Munck A, Craig J, Sermet I, Brownlee K, Schwarz M, Castellani C, Southern K. A European consensus for the evaluation and management of infants with an equivocal diagnosis following newborn screening for cystic fibrosis. J Cyst Fibros 2009;8:71-78

14. Brodlie M, Haq IJ , Roberts K, Elborn JS. Targeted therapies to improve CFTR function in cystic fibrosis. Gen Med 2015; 7:101-116

15. Temme R, Roggenbuck J, McNamara J. CF versus CRMS :Diagnostic Challenges in Cystic Fibrosis. Minn Med2012; $10: 42-44$

16. Megan N, Adamski C, Tluczeck A. Clinical practices for intermediate sweat tests following abnormal cystic fibrosis newborn screens. J Cyst Fibros 2011; 10: 460-465

17. Parra G, Bozzo R, Palomino MA. Fibrosis quística y enfermedad del CFTR: a propósito de un caso de traqueobroncopatía osteocondroplástica. Neumol Pediatr 2016;11:45-49 